\title{
La libertad a debate: la lectura de los textos cartesianos en Sartre y Ricœur
}

\author{
Freedom under discussion: Sartre's and Ricoeur's interpretation \\ of the Cartesian texts
}

ISABEL MORALES BENITO*

\begin{abstract}
Resumen: En el artículo se realiza una comparación sobre la libertad en Sartre y en Ricœur a partir de la lectura que ambos realizan de los textos cartesianos. En «La liberté cartésienne» (1947), Sartre sostiene que la libertad creadora que Descartes ve en Dios no es otra cosa que la auténtica libertad del hombre. En Lo voluntario y lo involuntario (1950), Ricœur determina a partir de la generosidad cartesiana que «querer no es crear». Se argumenta que, valorado en su conjunto, el planteamiento de Descartes se asemeja más a las tesis del consentimiento de Ricœur que a las del existencialismo de Sartre.

Palabras clave: Ricœur, Sartre, Descartes, libertad, generosidad, Dios.
\end{abstract}

\begin{abstract}
This paper carries out a comparison between Sartre's and Ricoeur's conceptions of freedom, based on their own interpretation of the Cartesian texts. In "La liberté cartésienne" (1947), Sartre argues that the creative freedom that Descartes observes in God is the genuine freedom of the human being. In Freedom and Nature (1950), Ricœur determines from the Cartesian generosity that "to will is not to create". This paper argues that Descartes' approach taken as a whole is more akin to Ricœur's consent approach than Sartre's existentialism theory.

Keywords: Ricoeur, Sartre, Descartes, Freedom, Generosity, God.
\end{abstract}

\section{Introducción}

Aun que sea de modo soslayado, es posible situar a Sartre como uno de los interlocutores a los que Ricœur se dirige en el momento de la elaboración de su tesis. El autor aparece poco en Lo voluntario y lo involuntario (1950): se hacen algunas menciones a los estudios fenomenológicos L'imagination (1936), L'imaginaire (1940), Esquisse d'une théorie des émotions (1939) y, menormente, a El ser y la nada (1943). No obstante, es posible plantear que Ricœur haya tenido presente a Sartre más de lo que parece por dos razones. La primera, porque en torno a estos mismos años encontramos dos escritos en los que sí analiza directamente su pensamiento: se trata de un comentario a la obra de teatro «Le Diable et le Bon

Recibido: 13/06/2018. Aceptado: 20/11/2018.

* Departamento de Humanidades, Universitat Internacional de Catalunya. Profesora de Antropología Filosófica. Pertenece al grupo de investigación SARX - Grup de Recerca en Antropologia de la Corporalitat (2017 SGR 031). Sus líneas de investigación son el pensamiento de Paul Ricœur, la libertad y la corporalidad, la Bioética y las Humanidades Médicas. Contacto: imorales@uic.es. 
Dieu», publicado en $1951^{1}$, y del artículo «Negatividad y afirmación original», publicado originalmente en 1956 (Ricœur1990, 295-316). Y la segunda, porque al examinar el contenido de Lo voluntario y lo involuntario, y en concreto lo referido a la libertad y su recepción de Descartes, es posible hallar un planteamiento diametralmente opuesto al que realiza Sartre. Esto permite afirmar que el conocimiento de Ricœur sobre este autor es tan real como lo es su deseo de presentar una alternativa al existencialismo nihilista. Dos son, asimismo, las ideas centrales que sustentan la comparación entre la libertad en Sartre y Ricœur: la de lo corporal como primer modo de contacto con la necesidad, y la de Dios, como referencia de una libertad absoluta que contrasta con la finitud humana.

Sobre Sartre, Ricœur afirma en su Autobiografía intelectual:

El Ser y la nada de Sartre sólo suscitó en mí una admiración lejana, pero ninguna convicción: ¿acaso un discípulo de Gabriel Marcel podía asignarle la dimensión de ser a la cosa inerte y no reservar sino la nada al sujeto vibrante de afirmaciones en todos los órdenes? (Ricœur 1997, 25).

Esta «ninguna convicción» constituye una pista valiosa que invita a preguntarse por qué las tesis de Sartre no convencen a Ricœur. La respuesta más evidente a tal cuestión se pone de manifiesto cuando Ricœur emplea la expresión «existencialismo negro» en Lo voluntario y lo involuntario y cuando dice de él que «no es, quizás, más que un idealismo decepcionado, y el sufrimiento de una conciencia que se ha creído divina y que se conoce ahora desgarrada» (Ricœur 1988, 50). Ricœur vincula aquí el «existencialismo negro» con un idealismo exacerbado, en que el yo se encuentra a sí mismo diferenciándose del cosmos y haciendo círculo consigo mismo. Aquí, dice, la conciencia es el producto del «rechazo irritado y, hasta cierto punto, enloquecido, [que] se eleva como desafío y como desprecio» (Ricœur 1988, 50). Ricœur levanta así un puente que une el idealismo y el existencialismo: en la medida en que la negación y el desprecio dejan de ser aspectos teoréticos y pasan a constituir el peso de la existencia humana concreta, el nihilismo se postula como actitud esencial ante la vida. Para Ricœur,

la relación estrecha entre el rechazo al que echa mano la libertad para armarse y la auto-posición de la conciencia explican sin duda suficientemente por qué una filosofía de la conciencia triunfante contiene en germen una filosofía de la desesperación. Basta con que el rechazo disimulado en el anhelo de auto-posición se conozca como rechazo para que la vanidad y el fracaso que lo constituyen se transformen rápidamente en desesperación con respecto a la pretensión de esta libertad titánica (Ricœur 1988, 510).

1 «Le Diable et le Bon Dieu»: aparecido por primera vez en Esprit (noviembre de 1951) y publicado también en Ricœur, P. (1999), en: Lectures 2. La contrée des philosophes, Paris: Seuil, pp. 137-148. Es significativo que en las primeras líneas de este escrito, Ricœur afirme que se haya sentido especialmente herido al ver la representación de la obra y que su argumentación consista en explicar el porqué de esta emoción. Puede observarse, así, una posición original de confrontación. 
Es esta una postura tan lejana al planteamiento de Ricœur como lo es el naturalismo, en el que el sujeto se pierde en favor del mundo de los objetos y de su movimiento mecánico. Como alternativa a estas posiciones, Ricœur parte en su investigación de la idea de cuerpo propio como primera condición de la existencia e incorpora, con ella, todo lo que denomina involuntario, ya sea relativo o absoluto. En esta categoría se incluyen tanto los motivos como los movimientos corporales, afectos, carácter, impulsos y condiciones generales de la existencia; todo lo que, de algún modo, podría considerarse como necesidad y que el idealismo rechaza por considerar que no es conciencia. Para Ricœur, lo que da razón a esta acogida es que todos estos elementos son de algún modo formas del cogito, porque no son necesidad pura sino necesidad vivida. El autor presenta, así, un cogito cuyos límites son más anchos que los que considera el idealismo (Ricœur 1988, 20-25).

Esta idea de cuerpo mostrada aquí es diferente para Sartre. Puede decirse que, en la medida en el existencialismo hace nacer la libertad de la conciencia desarraigada, Sartre desconoce lo corporal (Cladakis 2017, 186). De modo similar, haciendo referencia a Descartes y en virtud también de la idea totalitaria de elección, se sostiene que para Sartre no puede existir la unión entre el alma y el cuerpo que con tanto anhelo persiguió el autor moderno y a partir de la cual elaboró la teoría de las pasiones (Guenancia 2018). Sartre hace al sujeto responsable total de su existencia, lo que tiene como contrapartida identificar la libertad con la angustia. Ricœur, por el contrario, no ve posible una imputación pura, porque tal cosa excedería los límites de un cogito cuyo modo de ser inevitable es corporal, y plantea su idea de libertad más como una respuesta que como un decreto.

Este distinto modo de entender la libertad puede también formularse en términos de una libertad de creador y de una libertad de criatura. Este lenguaje, vinculado a las tesis de Descartes sobre la libertad de inferencia, permite ahondar en el debate y hallar en la raíz de los planteamientos una distinta recepción su pensamiento. En efecto, la diferente postura de los autores con respecto a Dios (recordemos que Sartre describe define su existencialismo como ateo (Sartre 1989, 27, 30), y que, por el contrario, Ricœur remite al inicio y al final de Lo voluntario y lo involuntario a la Trascendencia) conduce a conclusiones distintas: mientras Ricœur toma como inspiración la generosidad cartesiana, en la que el sujeto se humilla ante la necesidad, la verdad, y lo divino, Sartre aboga por aplicar al hombre lo que Descartes había apuntado que era divino y omnipotente, suplantando de este modo a Dios mismo ${ }^{2}$.

A partir de estas divergencias con respecto a Dios, no parece plausible que fuera el mismo Descartes el que leyeron Sartre y Ricœur. Es lícito, pues, preguntarse, como hace Grimaldi, si la recepción cartesiana de Sartre (y añadimos aquí la de Ricœur) se realiza en

2 Es posible, incluso, ahondar en la diferencia respecto a Dios acudiendo a una figura que está implícita en el pensamiento de los dos autores: se trata de Nietzsche. Diferentes autores han tratado separadamente sobre ello, y ponerlos en contacto confirma este dato. Por una parte, se afirma que, en cierto modo, Sartre es nietzscheano, pues comparte con él la idea de la muerte de Dios, aunque busque en la libertad y no en la fuerza o en la voluntad de poder los fundamentos de su moral inacabada [Bello, E. (2005), «La sombra de Nietzsche en Sartre», Daimon n 35 , pp. 41-62]. También parece claro que la fundamentación de la libertad creadora de Sartre a partir de la negación de Descartes se encuentra en la nietzschena muerte de Dios [Zima, P. V. (2015), Subjectivity and Identity: Between Modernity and Postmodernity, London, New York: Bloomsbury, p. 93]. Por otra parte, y situándose en una posición antagónica, se presenta a Ricœur como un pensador antinihilista porque toda su obra, y particularmente en el inicio, está marcada por el sí del consentimiento [Abel, O. (2010), «L'adolescence du consentement», en: J. Poreé y G. Vincent (eds.): Paul Ricœur. La pensée en dialogue, Rennes: PUR, pp. 105-114]. 
forma de continuidad o de interpretación (Grimaldi 1987, 67-88). Para responder a ello, en este trabajo se examina, en primer lugar, el planteamiento del pensador existencialista acerca de la conciencia como auto-posición absoluta y la justificación que halla en Descartes para afirmar que la libertad del hombre es creación pura. En segundo lugar, se traen a colación las aportaciones de Descartes acerca de la atención, de las semillas de verdad y de la generosi$\mathrm{dad}$, nociones que Ricœur incorpora en su análisis y prolonga hasta formular su tesis sobre el consentimiento. El resultado de la comparación revela la común problemática de Descartes y de Ricœur de reconciliar la libertad y la finitud humana pero una distinta solución.

\section{Una lectura cartesiana: Sartre y la libertad creadora}

\subsection{Ser para-sí y libertad como distanciamiento}

Para Sartre, la conciencia y la negación se encuentran estrechamente vinculadas, hasta el punto de que el hombre encuentra su forma idónea de expresión en el nihilismo. Sartre identifica la conciencia del hombre con el ser para-sí y lo presenta como un ser cuya característica fundamental consiste en la carencia, oponiéndolo al ser en-sí de las cosas, cuyo rasgo es la plenitud, lo lleno, lo macizo (Sartre 1993, 35-36). Las cosas son algo, tienen una esencia concreta y determinada. La conciencia, por el contrario, es la que indica que existe una no-coincidencia consigo mismo. «La realidad humana -afirma Sartre-, por la cual la carencia aparece en el mundo, debe ser a su vez una carencia» (Sartre 1993, 120).

Que la conciencia sea "para” sí significa que no está "en” las cosas, sino que su relación consigo misma pasa por un momento de trascendencia de las propias cosas: el sí no tiene entidad, sino no podría ejercer este movimiento de vuelta hacia sí mismo. Lo que distingue, pues, la conciencia de las cosas no es un ser diferente, sino no-ser todas ellas: es la negación, la vacuidad y la separación lo que la hace capaz de saberse diferente del ser en-sí.

Es en esta nada del para-sí donde Sartre encuentra el lugar para la libertad del hombre, porque ser libre significa ser capaz de todo, y para ser capaz de todo es necesario no ser previamente nada. Por el hombre, en efecto, la nada entra en el mundo, y lo hace en forma de libertad, siendo la conciencia una apertura sin límites hacia el futuro y hacia lo nuevo. Lo posible se configura de este modo como una estructura del para-sí (Sartre 1993, 129-135), y es esta estructura la que Sartre ve prefigurada en la duda de Descartes, porque dudar es «nadificar todo lo que existe» (Sartre 1947, 326), ponerlo entre paréntesis, y es este ejercicio el que permite al hombre separarse del mundo y contemplarlo desde lo alto:

La realidad humana no puede sustraerse al mundo -en la interrogación, la duda metódica, la duda escéptica, la epojé, etc.- a menos que sea, por naturaleza, arrancamiento de sí misma. Es lo que [vio] Descartes, quien funda la duda sobre la libertad, reclamando para nosotros la posibilidad de suspender nuestros juicios (Sartre 1993, 61).

Para Sartre, nadie antes que Descartes había puesto el acento en el vínculo entre el libre arbitrio y la negatividad (Sartre 1974, 327). En la finitud del hombre, es decir, en su capacidad de no-ser, encuentra Sartre el origen de la libertad: siendo capaz de equivocarme descubro que no estoy atado a las verdades, sino que puedo dudar de ellas, y hacerlo volunta- 
riamente. Asimismo, si ser hombre -ser para-sí-, es separarse, y en esto consiste su libertad, esta misma consiste en un acto de aislamiento, de un modo similar al que plantearon los estoicos (Sartre 1993, 60). Así pues, la libertad consiste en hacerse a uno mismo a partir de la nada. Para Sartre, ser hombre consiste en una existencia que precede a la esencia, lo que significa «que el hombre empieza por existir, se encuentra, surge en el mundo y que después se define» (Sartre 1989, 17).

De tal modo, se plantea así una voluntad pura y total. Lo que define al hombre es su radical distinción con el mundo y, por ello, con la necesidad. El hombre nace, pues, siendo exilio, y su voluntad se revela como una capacidad de serlo todo:

Si en efecto la existencia precede a la esencia, no se podrá jamás explicar por referencia a una naturaleza humana dada y fija; dicho de otro modo, no hay determinismo, el hombre es libre, el hombre es libertad (Sartre 1989, 26).

La libertad aparece pues, como una exigencia y como un deber. Elegir no es un capricho, sino una condena (Sartre 1989, 48). El hombre no descubre dentro de sí nada dado, nada hecho; no descubre valores, sino que los crea. En este sentido, el existencialismo de Sartre no se postula como un quietismo, sino todo lo contrario: el hombre es acción, y además el alcance de esta acción es universal. En cada acto libre, en cada decisión, está en juego no solo la propia vida sino toda la humanidad, debido a que la subjetividad, por ser un para-sí es también un ser para-otro (Sartre 1993, 250ss). Este vértigo es el que conduce al hombre cobarde a refugiarse en lo que Sartre llama la «mala fe», que consiste en engañarse a sí mismo y volverse cosa aceptando unos supuestos valores o ética establecida, formulada de acuerdo con una supuesta naturaleza humana ${ }^{3}$.

\subsection{Dos libertades: la de autonomía y la de productividad}

Se acaba de ver que una de las claves para comprender el pensamiento de Sartre es el vínculo entre la negación y la libertad. Otro punto esencial de su planteamiento es la desvinculación entre la verdad y la libertad. La esencia del planteamiento es que, si el hombre debe suscribir las verdades que su entendimiento conoce, entonces no es libre, pues está «obligado» a reconocerlas como tales. Lo sorprendente de esta posición reside en que, nuevamente, es a Descartes a quien remite Sartre, haciendo una particular interpretación de sus textos referidos al error, a la libertad y a Dios, en especial los de la cuarta meditación metafísica, en la que se identifica como causa del error la no concordancia entre el entendimiento, que es finito, y la voluntad, que es infinita (Descartes 2011, 185-188).

Para desarrollar este argumento, Sartre distingue en el pensamiento cartesiano dos dimensiones o tipos de libertad: la de autonomía y la de productividad, y afirma que nuestra concepción moderna de la libertad está basada en la primera concepción. Tal es lo que queda expresado en su célebre afirmación:

3 Los ejemplos de la mujer que acude a la primera cita y del camarero son al respecto muy ilustrativos (Ibíd. pp. 29-101). 
Nosotros, franceses, que vivimos desde hace tres siglos en la libertad cartesiana, nosotros entendemos implícitamente por "libre arbitrio" el ejercicio en un pensamiento independiente más que la producción de un acto creador (Sartre 1974, 315).

He aquí la tesis central que propone Sartre: Descartes avanzó en su pensamiento aquello en lo que consistía la verdadera libertad -un acto creador, como el de Dios-, pero ni él ni el mundo moderno, hasta la llegada del existencialismo, se atrevieron a asumirlo hasta sus últimas consecuencias y se resignaron a vivir en lo que sería el primer grado (Sartre 1974, 332s).

La identificación del libre albedrío con la autonomía se fundamenta, a juicio de Sartre, en el ejercicio de negación y de la duda: somos capaces de no querer aquello que conocemos y de crear un método que nos conduzca a la verdad. Para Descartes, la voluntad es, por tanto, una facultad infinita, en la que observamos la huella más firme de Dios en nuestras almas (Descartes 2011, 186), y esta libertad se encamina hacia el conocimiento de la verdad. La duda de Descartes es, en este sentido, provisional y metódica. No obstante, a pesar de que la voluntad y la libertad tienen una extensión más amplia que la del conocimiento, el hombre es un ser finito, de lo que se deduce que su libertad es fundamentalmente una libertad que no es indiferente, sino que es relativa a las verdades que conoce (Descartes 2011, 186s).

Sartre acoge la identificación cartesiana entre negación y libertad. Sin embargo, es precisamente la idea acerca de la no indiferencia de la voluntad donde se muestra discordante: si nuestra voluntad está determinada a aceptar las verdades que clara y distintamente se le presentan, ¿no está entonces, de algún modo, totalmente coaccionada? Como indica Grimaldi, es preciso poner atención en la sutil interpretación que realiza Sartre cuando traduce a Descartes: allá donde el filósofo moderno habla de "propensio", haciendo referencia a la fuerza con que la verdad aparece, el existencialista habla de «la volonté contrainte d'affirmer» (Sartre 1974, 302), es decir, de una «voluntad constreñida [coaccionada, sometida, obligada] a afirmar» (Grimaldi 1987, 72). De acuerdo con esta lectura de Sartre, la voluntad está privada de toda iniciativa, pues ya provenga de la luz natural o de la revelación de Dios, las verdades adquieren categoría de ley ante el entendimiento, que no puede sino inclinarse ante ellas. Sartre concluye, pues, que según este planteamiento, el hombre no es libre más que nominalmente (Sartre 1974, 330). La presunta experiencia de autonomía que la duda le otorga al hombre no es más que la experiencia oculta de una dependencia mayor. La libertad no es, entonces, verdadera libertad, ya que aunque es autónoma, esta no es creadora. Nos encontramos, pues, ante un engaño. Tal y como explica,

Descartes oscila perpetuamente entre la identificación de la libertad con la negatividad o negación del ser -lo que sería la libertad de indiferencia- y la concepción del libre arbitrio como simple negación de la negación. En una palabra, le ha faltado concebir la negatividad como productiva. Extraña libertad: (...) ésta se descompone en dos tiempos: en el primero, es negativa y autónoma, pero se reduce a rechazar nuestro consentimiento al error o a los pensamientos confusos; en el segundo, ésta cambia de significación, es adhesión positiva, pero entonces la voluntad pierde su autonomía y la gran claridad que está en el entendimiento penetra y determina la voluntad. ¿Es esto lo que Descartes quería? La teoría que él ha construido, ¿responde 
verdaderamente al sentimiento primero que este hombre independiente y orgulloso tenía de su libre arbitrio? No lo parece (Sartre 1974, 328) ${ }^{4}$.

La verdadera libertad es, entonces, para Sartre, aquella que es productiva y que Descartes puso en Dios, pero que, por convencionalismos de la época, el filósofo moderno no pudo desarrollar en el hombre (Sartre 1974, 330). «Serán necesarios -dice- dos siglos de crisis (...) para que el hombre recupere esta libertad creadora que Descartes puso en Dios» (Sartre 1974, 334s). Es ésta una libertad que no se somete al bien o a la verdad, sino que lo crea: el Dios de Descartes crea por decreto; su ser infinito no está sujeto a cualquier norma lógica o moral ni está limitado, por ejemplo, por la cuadratura del cuadrado o por la circularidad del círculo. Si un círculo tiene tal forma, la tiene porque Dios lo ha querido así, de la misma manera que podría haberlo querido de otra forma. Dicho de otro modo: las cosas no son buenas o verdaderas porque lo sean en sí mismas, según lo cual incluso Dios estaría sometido a ellas, sino que son buenas porque Dios, que las ha creado, lo ha querido así. «Es la libertad la que es el fundamento de la verdad, [porque] Dios ha inventado el bien» (Sartre 1974, 333)5. Para Sartre, concebir, por un lado, un Dios infinito y, por otro, que éste esté sometido a las reglas de lo creado sería incurrir en una contradicción de términos. Esta es la libertad de indiferencia de Dios que debe ser, para Sartre, la libertad humana.

Al final, Sartre concluye que la verdadera exigencia de la primera libertad -la de autonomía- lleva hasta los confines de la segunda -la de productividad-. La negación y el poder que le confiere al hombre la duda deben llegar hasta los límites de no estar sometido a una verdad, sino a crearla. Para Sartre, pues, no existen distintos grados o dimensiones de libertad: «la libertad es una» (Sartre 1974, 314), tal es la afirmación con la que Sartre inicia su escrito sobre la libertad cartesiana. Es una porque es total, absoluta, porque no admite grados (Sartre 1974, 318), y esto es así porque el hombre es enteramente un para-sí: todo él es por oposición a las cosas, todo él es no-ser o lo que está por venir, por lo tanto, todo él es libertad. Una libertad a medias no sería, pues, para Sartre, una libertad.

\section{Otra lectura cartesiana: Ricour y la generosidad}

El planteamiento de Ricœur con respecto a la libertad difiere del de Sartre tanto en la conclusión como en los distintos argumentos que lo sustentan. Ricœur apunta en Lo voluntario y lo involuntario la idea de consentimiento como clave para vincular el Cogito a la necesidad, y lo hace basándose en la idea de generosidad cartesiana, de ahí que su lectura de Descartes sea particularmente distinta.

La divergencia de Ricœur con Sartre parte de la noción de nada, que para Ricœur resulta singularmente sospechosa: «la idea de la nada-explica en 1950- es una fuente inagotable de equivocaciones» (Ricœur 1988, 36), la más fundamental de las cuales es la del propio yo y de su libertad. Pocos años más adelante, esta crítica se explicita detalladamente:

4 Sartre argumenta que este mismo engaño es el que sucede, por ejemplo, al aprender matemáticas o al caminar (cfr. Sartre 1974, 316, 322, 327).

5 La cursiva es mía. 
Es su noción [la de Sartre] de ser en sí la que le sirve para cincelar su noción de la nada, que es demasiado pobre y ya cosificada. (...) Todo lo que ha demostrado es que para ser libre hay que constituirse en no-cosa; pero no-cosa no es no-ser: nothing is not no-being. Este es, en mi opinión, el punto delicado de su filosofía; su filosofía de la nada es la consecuencia de una filosofía insuficiente del ser (Ricœur 1990, 312).

Si Sartre parte de una concepción unívoca del ser (o es o no-es) que conduce a una libertad planteada en términos radicales (o se es libre totalmente -divinamente- o se es "cosa", subyugado a la necesidad), Ricœur parte de un cogito ensanchado e intenta explorar las regiones intermedias de la subjetividad en las que el yo, sin perder su originalidad y distinción con respecto a las cosas del cosmos, ejerce su libertad de criatura por medio del acto del consentimiento.

Fieles a nuestra regla metódica -apunta Ricœur-, suspendemos aquí las ideas y venidas de la conciencia fascinada por el Sí mismo y por la Nada, y concebimos una afirmación de sí, presta a la reflexión, que es la llave común del amor inocente hacia mí mismo y de esta conciencia de sí fascinada (Ricœur 1988, 75).

Esta afirmación es la que Ricœur ve prefigurada de alguna manera en Descartes: el filósofo de la modernidad, dice, «nos invita, más de lo que él lo ha sospechado, a cambiar de régimen de pensamiento» (Ricœur 1988, 27) y sus tres máximas formuladas a Isabel de Bohemia en la carta del 4 de agosto de 1645 sirven al autor como inspiración para estructurar su eidética de la voluntad a partir de la triple distinción de la decisión, del esfuerzo y del consentimiento (Ricœur 1988,529). Muy atento al Tratado de las pasiones y a su correspondencia, Ricœur toma de Descartes toma la idea de generosidad que remite a una «conciencia considerada en su impulso hacia lo otro» (Ricœur 1988, 75), dando un salto hacia adelante; una conciencia que es opuesta a la conciencia desarraigada que plantea Sartre. Antes de examinar la noción de generosidad, es preciso detenerse para analizar la idea de la atención y la de semillas de verdad. Ambas nociones preparan el terreno de la generosidad y son tomadas por Ricœur para darles forma en su estudio sobre el querer ${ }^{6}$.

\subsection{La atención y las semillas de verdad}

Por la atención, Descartes muestra que la voluntad mantiene una relación de autonomía con respecto al entendimiento. La verdad no se impone forzosamente a la voluntad, como piensa Sartre, sino que aparece solamente si la voluntad consiente en que aparezca, esto es, cuando se dispone libremente a recibirla, a aceptarla y a retenerla. La atención depende enteramente de nuestra voluntad, por eso, la verdad que recibimos es captada solamente cuando queremos captarla. Incluso, de acuerdo con la segunda regla de la moral provisional que plantea Descartes, somos capaces de querer firme y resolutamente algo de lo que todavía no

6 Para un desarrollo de la noción de atención en Ricœur, cfr. el estudio que presentó en 1939 en el Cercle philosophique de l'Ouest, recogido en: Ricœur, P. (2013), «Étude phénoménologique de l'attention et de ses connexions philosophiques», en: Anthropologie philosophique. Écrits et conférences 3, Paris: Seuil, pp. 51-93. 
tenemos una certeza cognoscitiva. En la tercera regla, además, Descartes hace hincapié en la capacidad de autonomía que tenemos con respecto a nuestros deseos y a aquello que conocemos, tal y como muestran los estoicos. En esto, precisamente, se distinguen los hombres y la beatitud que estos alcanzan, que es proporcional al grado de libertad que la capacidad de autodominio y abnegación les otorga (Descartes 2011, 87-89). A juicio de Grimaldi (1987, 73), la carta de Descartes a Mesland del 9 de febrero de 1645 -carta que Sartre obvia en su argumentación- justifica una interpretación no determinista del estatuto de la voluntad respecto del entendimiento porque, según ésta, podemos abstenernos «de admitir una verdad evidente» (Descartes 1972, 173) y porque «la libre disposición de nuestras voluntades nos pertenece realmente» (Descartes 1997, artículo 153, 226-227).

Así pues, es necesario comprender los matices del carácter «invencible», «irresistible» o «determinante» con que la verdad se nos aparece; ésta nos «atrapa», pero lo hace solamente si somos nosotros quienes nos entregamos libremente a ella y quienes, por eso, nos adherimos (Grimaldi 1987, 81s). La verdad, por tanto, sólo tiene un carácter coercitivo si somos nosotros quienes se lo otorgamos. La búsqueda infatigable de un método que nos conduzca a la verdad y la determinación para su uso constante no es, sino, otra cosa que una manifestación de esta independencia de la voluntad. Si fuésemos, además, absolutamente indiferentes a las verdades que conocemos significaría que somos incapaces de ella, lo que desdice del planteamiento de Descartes.

Con respecto a las semillas de verdad (Descartes 2011, 120), lo primero que cabe preguntarse es por qué la libertad de indiferencia en el hombre es su grado más bajo de libertad. O, planteada la pregunta en sentido inverso, por qué el conocimiento de la verdad nos hace más libres. La noción de «semillas de verdad», tan «constante como precoz» en el pensamiento de Descartes (Grimaldi 1987, 82s), responde bien a estas cuestiones: la verdad está contenida por las ideas innatas en el conocimiento a modo de semillas, de la misma manera que el fuego está contenido en el sílex. En este sentido, la verdad es connatural al hombre, y en su hallazgo y conformidad encuentra su pleno desarrollo. Tal es lo que nos hace semejantes a Dios. Así pues, la verdad no es lo opuesto a la voluntad o a la libertad, tal y como plantea Sartre; no hay una oposición radical o una alteridad absoluta entre el no-ser del hombre y el ser de la verdad, sino que el conocimiento del hombre se muestra más bien como un todavía no-ser. La verdad, pues, se revela como el desenvolvimiento y la plenitud de una voluntad y de un entendimiento que buscan lo que no tienen, porque no son infinitos ni perfectos ${ }^{7}$.

He aquí, pues, algunas diferencias principales entre Descartes y Sartre que pueden deducirse a partir de la comparación: mientras el primero fundamenta todo su planteamiento en la verdad, el segundo la rechaza de pleno. Para Descartes, la búsqueda de la verdad es la constante que aparece en toda su obra; la confianza en que esta existe, en que el hombre es capaz de ella y que de su encuentro depende nuestra beatitud puede encontrarse expresada de muy distintos modos. Para Sartre, en cambio, la verdad del hombre no existe, lo que hace que su sistema sea

7 Esta misma idea es la que defiende Kaposi en su lectura de Descartes cuando afirma que la libertad del hombre es la de la no-indiferencia. Para el autor, la magna propensio es la más grande libertad, hasta el punto de que cuando no se tienen motivos o verdades evidentes que aceptar, el más recto uso de la libertad es el de abstener el juicio [Kaposi, D.,(2004), «Indifférence et liberté humaine chez Descartes», Revue de métaphysique et de morale 41, n. ${ }^{\circ} 1$, pp. 85-86]. 
incompatible de suyo con las nociones de atención y semillas de verdad (Grimaldi 1987, 84), porque no hay naturaleza en el hombre ni verdad que pueda vencer su capacidad de serlo todo.

\subsection{La generosidad cartesiana}

Para Descartes, el hombre experimenta una suerte de satisfacción en la aceptación de las verdades, e incluso en la abnegación estoica frente a los deseos. En la indigencia del conocimiento, por tanto, el hombre experimenta una suerte de infinito y una beatitud natural; somos capaces de realizar el bien aunque seamos incapaces de juzgar. Tal es la experiencia de la generosidad, que es la libertad del hombre: capacidad para decir no pero también capacidad de decir sí y acoger las verdades para las que está dispuesto (Armour y Johnston 1997, 695-710). Esta libertad, por tanto, no es una demostración sino sobre todo un ejercicio. Descartes distingue, al respecto, entre las almas bajas o vulgares y las almas más elevadas ${ }^{8}$, que también llama las más fuertes o generosas ${ }^{9}$. Esta distinción supone reconocer la existencia de una «naturaleza individual constituida» (Grimaldi 1987, 86), según la cual unos hombres pueden ser más generosos que otros o, lo que es lo mismo, querer más o menos la infinitud de su voluntad. Todos los hombres poseen la misma libertad, en efecto, pero no todos son capaces de querer y alcanzar los bienes del alma, por lo que no todos son capaces de ejercitarla del mismo modo.

El hombre generoso es aquel que, como reconoce Sartre, realiza su libertad en un primer momento que es negación y autonomía, pero esta negación la ejercita también consigo mismo, y lo hace en función de los bienes que conoce, que alcanza y que acepta. Y lo hace, además, conociendo su propia finitud. La libertad del hombre pasa, entonces, por la asunción de las verdades que vienen de Dios, verdades eternas que el hombre no decide por decreto, sino que asume, encontrándose como consecuencia a sí mismo. La negatividad de la libertad del hombre, por tanto, es subsidiaria de una positividad más fundamental: la de Dios y la que, por Él, el hombre es capaz de ejercitar.

\subsection{Ricœur y el consentimiento}

Lo que para Descartes es generosidad, para Ricœur es consentimiento, que es el último momento eidético de la voluntad, en el que ésta se encuentra con la necesidad absoluta. «El querer -dice Ricœur- es ante todo impulso, lanzamiento, salto, es decir, "generosidad"» (Ricœur 1988, 76). Ricœur distingue claramente dos aspectos de la libertad: «una cosa, según parece, es la libertad de elección y de moción», en la que la voluntad se encuentra con el involuntario relativo de los motivos y de los poderes corporales, «y otra cosa, la libertad de consentimiento», en la que la voluntad se encuentra con el involuntario absoluto del carácter, el inconsciente y la vida (Ricœur 1988, 528).

8 Cfr. Carta a Isabel de Bohemia del 18 de mayo de 1645 [Descartes, R. (1999), Correspondencia con Isabel de Bohemia y otras cartas, Barcelona: Alba, p. 63]; la carta del 21 de julio de 1645 (ibíd., p. 79) y los artículos 48 y 49 del Tratado de las pasiones [Descartes, R. (1997), Las pasiones del alma, op. cit., pp. 125-126].

9 Carta a Isabel de Bohemia del 6 de octubre de 1645. En ella Descartes explica que la opinión que debemos tener sobre nosotros mismos versa sobre el equilibrio entre la vanidad y la indigencia [Descartes, R. (1999), Correspondencia..., op. cit., p. 113] y que la felicidad es compatible con la imperfección (ibíd., p. 111). 
Podría parecer que el primer aspecto es equitativo al de la libertad de autonomía que señala Sartre. No obstante, lo que separa a los autores en este caso no es solamente una consideración de grados, sino de esencia: mientras Sartre niega la necesidad en un acto total y unitario, Ricœur solamente la niega en un primer momento, que es transitorio, y que debe ser superado por el acto de afirmación o de consentimiento final (Ricœur 1990 315). En la decisión y en la moción, Ricœur describe cómo la relación del sujeto con la necesidad es la de circularidad: el motivo funda, legitima y justifica la decisión (Ricœur 1988, 80), y sólo por ésta, el motivo adquiere la categoría de lo que es. En otras palabras: como ocurre gracias a la independencia de la voluntad cartesiana y su aceptación de las verdades, la voluntad decide y se determina a sí misma a partir de lo que acoge y decide que la determine. De acuerdo con el planteamiento de Descartes, es ésta una libertad que no es indiferente, ya que nace como respuesta a lo que es recibido. Pero esta respuesta es activa, libre, atenta, porque es por este acto de fijación y afirmación expreso de la voluntad por lo que la decisión es tomada y la moción es realizada.

Es en el tercer momento de la voluntad, el del consentimiento, cuando Ricœur lleva hasta los límites la tesis de la generosidad cartesiana y la afirmación última. Si antes los motivos y los poderes corporales eran lo que eran gracias al acto recíproco de la voluntad, que les otorga un sentido y los conduce de una forma genuina y novedosa, lo involuntario absoluto es aquello que llega ante la voluntad sin posibilidad de cambio ni de respuesta. Ante el carácter, el inconsciente y la vida, las dos únicas dos salidas posibles son su rechazo absoluto o su acogimiento de lo que viene dado como tal.

Y es en este punto, en el de la relación del sujeto con la necesidad, donde Ricœur deja su impronta. En el momento del encuentro con la necesidad surge una primera reacción de negación o de rechazo: yo no soy la necesidad, afirma el yo en un acto de reafirmación de sí mismo. En este instante, es posible apreciar una doble negación: la padecida y la querida. La primera es la que ejerce la necesidad para con el sujeto, por el que lo anula; la segunda es la respuesta que formula el yo frente a este "ataque" y que se materializa en forma de rechazo y de exilio. El camino del consentimiento que plantea Ricœur debe necesariamente pasar por estos dos estadios, pero no para instalarse en ellos, como hacen el naturalismo (Ricœur 1988, 21) y el idealismo, sino para superarlos (Ricœur 1988, 489). La detención en la segunda negación conduce al exilio del mundo que proclama el «existencialismo negro», en el que el sujeto se aboca a la nada y se anula a sí mismo. Para Ricœur, el momento de negación no puede ser último ni el fundamental. El momento de trasgresión del punto de vista conduce, finalmente, a «descubrir la afirmación original en esa negación de la negación» (Ricœur 1990, 315). Como apunta Scott-Baumann (2009, 29), este no es el camino que toma Sartre, para quien la negación se convierte en un fin en sí mismo.

Trascender esta doble negación es lo mismo que "saltar hacia adelante" en un acto de generoso consentimiento, que consiste en decir sí a la necesidad:

La generosidad que Descartes señala carece de angustia -explica Ricœur-. La oposición de mi ser al ser de las cosas, que viene a reforzar con un poderoso contraste esta seguridad de poder-ser por mí mismo, puede permanecer perfectamente en la tonalidad alegre evocada por Descartes en Tratado de las Pasiones y en su Correspondencia (Ricœur 1988, 77s). 
La libertad, por tanto, comienza «inaugurando el ser», pero termina «subordinándose a la iniciativa de las cosas», no osando, sino consintiendo y rindiéndose (Ricœur 1988, 528). La libertad «no es un acto puro, es en cada uno de sus momentos actividad y receptividad; se hace acogiendo lo que no hace: valores, poderes y pura naturaleza» (Ricœur 1988, 530).

\section{Conclusiones}

La comparación establecida entre la libertad en Ricœur y en Sartre a propósito de su recepción de Descartes permite concluir que la lectura de Ricœur se aproxima más al pensamiento de Descartes que la que realiza Sartre, de tal modo que, respondiendo a la cuestión planteada al inicio, puede afirmarse que el primer autor realiza un ejercicio de continuidad, mientras que el segundo surge más bien de una interpretación. Se presentan a continuación cinco conclusiones que sintetizan lo argumentado y cierran el debate. La primera de ellas refiere los temas cartesianos que resultan ineludibles para comprender su planteamiento sobre la libertad. La segunda, tercera, cuarta y quinta permiten, asimismo, plantear a grandes rasgos cuál es la planteamiento que tienen Sartre y Ricœur sobre la libertad y conocer de qué modo resuelven el conflicto entre esta misma y la finitud humana.

1 - Deben considerarse como argumentos irrenunciables en Descartes: 1) La provisionalidad de la duda y el ejercicio de independencia por medio de la negación como un momento pasajero, superado por el encuentro con la verdad, y su aceptación y afirmación. 2) El intento de Descartes de reconciliar lo anímico y lo corporal y su acogida del cuerpo en sus trabajos sobre las pasiones. 3) Que Descartes no niega a Dios, por el contario, tanto en lo relativo al conocimiento como a la moral y a la libertad, sitúa al hombre como criatura dependiente y plantea la beatitud más como un acto de conformidad que de rebeldía. A pesar de que para Descartes la instancia de la voluntad es lo que conecta al hombre con Dios, el filósofo se mantiene en la opinión de que el hombre es criatura y, por tanto, su voluntad no es de indiferencia, aunque sea infinita. Según se ha visto, en «La liberté cartésienne» Sartre no parece tener en cuenta estos tres elementos, ni tampoco considerar la intención global del planteamiento de Descartes. Por el contrario, Sartre se sirve de argumentos cartesianos que son reales pero que son parciales, desconectados de lo que parece ser la intención final de Descartes de describir a un hombre feliz en su finitud. Estos tres elementos, por el contrario, están recogidos en Lo voluntario y lo involuntario de Ricœur y se hallan en el trasfondo de toda la obra, que cristaliza en la tesis del consentimiento.

2 - La libertad que conciben Sartre, por un lado, y Ricœur y Descartes, por otro, difiere en que el primero la considera una, por lo que o es total o no es, mientras que los segundos, sin negar la autenticidad de la libertad humana, la entienden de un modo gradual y no absoluto. Concebir la libertad como total conduce a entender al hombre como un exilio del mundo, de la naturaleza, y por confrontación a la necesidad, y tal es el punto que intenta evitar Ricœur en su obra. Para Sartre, en efecto, las propuestas que realiza Ricœur no pueden ser sino nociones extrañas en su filosofía: un querer motivado; una conciencia que descubre -no crea- los valores; una voluntad que es recíproca de lo involuntario; una libertad que es en último término consentimiento; y un cogito ampliado hasta los confines de la necesidad son contradicciones, términos irreconciliables desde el punto de vista de 
la lógica sartreana. Pero para Ricœur, lo que es una oposición para la comprensión, es un hecho para la vida, que resulta paradójica y misteriosa por cuanto ata la libertad y la necesidad con antelación a la distinción mental que hacemos de ellas. Tal como explica, la unión está operada más acá del entendimiento. Para Ricœur, la libertad que plantea Sartre, precisamente por nacer de la conciencia, es una libertad que, en el fondo, es ideal y no real. Se trata de una libertad que permanece solamente en el imaginario de aquel que piensa acerca de ella; una libertad «matemática» que poco tiene que ver con la experiencia.

3 - Tanto Sartre como Ricœur y Descartes vinculan la libertad con la infinitud, por lo que remiten a Dios en sus argumentos. No obstante, el modo en que conciben esta infinitud es distinto: para los segundos, la cualidad de infinitud no reside en el poder de creación de la verdad sino en la generosidad que experimenta el hombre beato. En el consentimiento de la verdad y de la finitud -esto es, en la adecuación de la voluntad al entendimiento-, el hombre ejercita el máximo grado de su libertad, no negando ni nadificando su naturaleza humana, sino afirmándola y acogiéndola, tal como plantea Ricœur. El hombre pues, sin renunciar a su finitud, es capaz de lo infinito. Es así como «la indiferencia y la libertad pueden ser semejantes en el hombre y en Dios» (Grimaldi, 1987, 88), porque ni la una ni la otra son unívocas. Si la indiferencia de Dios es la señal de su perfección, en el hombre lo es de su imperfección. Ante esta paradoja, los autores dan una solución dispar: mientras Sartre opta por negar la existencia de la verdad, Descartes y Ricœur optan por reconocer el estado de ignorancia. Para estos últimos, la libertad no puede ser una libertad hacia la nada, sino que, por la naturaleza del hombre, está destinada a hacerse una con la verdad, pues solo en ella encuentra su plenitud.

4 - Como se ha dicho, la filosofía de Sartre aboca al hombre hacia la nada. Tal es el precio de comprenderlo separado de la necesidad: identificarlo con la negación de sí mismo y del mundo. Pensando su planteamiento como un antídoto contra la naturalización de la conciencia, Sartre despoja al hombre de naturaleza, anulando con ello la esencia humana. Frente a ello, la solución que plantea Ricœur, inspirado en Descartes, se sitúa como un punto intermedio, como una conciliación entre el naturalismo y el exilio: decir sí a la necesidad es decir sí al cogito diciendo a la vez sí a Dios. Ricœur no obvia las paradojas del planteamiento, y menos aún de la experiencia ambivalente de la libertad humana, pero, lejos de querer eliminarla, la acoge como donante de sentido. La antropología que plantea Ricœur a través de su eidética de la voluntad se encamina, por tanto, no a la nada, sino a la afirmación, a la dotación de sentido. Así, de la misma manera que Descartes ve en la acogida de la verdad una plenitud del alma, Ricœur ve en el consentimiento de la necesidad el primer modo - no definitivo- de decir sí a la humanidad del hombre; pero de un hombre que se reconoce como tal y asume su condición de no ser Dios.

5 - La generosidad y el consentimiento se plantean, al fin, como un correctivo de la vanidad de la conciencia y como una humilde afirmación de sí que conduce a no saberse rechazada del mundo, sino acogida por él. La libertad, entonces, ya no es angustia, sino estima de sí. Las últimas palabras de Lo voluntario y lo involuntario son una respuesta firme a un Sartre que pretende una libertad titánica: «querer no es crear» (Ricœur 1988, 532), dice Ricœur. Querer es, más bien, saberse determinado por la necesidad y aceptar esta determinación, determinándose a sí mismo en ese acto. Querer humanamente es, en una palabra, consentir. 


\section{Referencias}

Abel, Olivier (2010), «L'adolescence du consentement», en: J. Poreé y G. Vincent (eds.): Paul Ricoeur. La pensée en dialogue, Rennes: PUR, pp. 105-114.

Armour, Leslie y Johnston, Suzie (1997), «Ipséité et générosité selon Descartes», Laval théologique et philosophique n⿳553 (3), pp. 695-710.

Bello, Eduardo (2005), «La sombra de Nietzsche en Sartre», Daimon n ${ }^{\circ} 35$, pp. 41-62.

Cladakis, Maximiliano Basilio (2017), «Negatividad e intersubjetividad. La dimensión originaria del conflicto en Kojève y en Sartre», Ideas y Valores 66 nº165, pp. 171-189.

Descartes, René (2011), Discurso del método y Meditaciones metafísicas, Madrid: Tecnos.

Descartes, René (1999), Correspondencia con Isabel de Bohemia y otras cartas, Barcelona: Alba.

Descartes, René (1997), Las pasiones del alma, Madrid: Tecnos.

Descartes, René (1972), Oeuvres de Descartes (edición de Charles Adam \& Paul Tannery), IV, Paris: Vrin.

Grimaldi, Nicolas (1987), «Sartre et la liberté cartésienne», Revue de Métaphysique et de Morale $\mathrm{n}^{\circ}$ 92, pp. 67-88.

Guenancia, Pierre (2018), La voie de la conscience. Husserl, Sartre, Merleau-Ponty, Ricour, Paris: PUF.

Kaposi, Dorottya (2004), «Indifférence et liberté humaine chez Descartes», Revue de métaphysique et de morale 41, n.o 1, pp. 73-99.

Ricœur, Paul (2013), «Étude phénoménologique de l'attention et de ses connexions philosophiques», en: Anthropologie philosophique. Écrits et conférences 3, Paris: Seuil, pp. 51-93.

Ricœur, Paul (1999), «Le Diable et le Bon Dieu», en: Lectures 2. La contrée des philosophes, Paris: Seuil, pp. 137-148.

Ricœur, Paul (1997), Autobiografía intelectual, Buenos Aires: Nueva Visión.

Ricœur, Paul (1990), «Negatividad y afirmación original», en: Historia y verdad, Madrid: Encuentro, pp. 295-316.

Ricœur, Paul (1988), Lo voluntario y lo involuntario, Buenos Aires: Docencia.

Sartre, Jean Paul (1993), El ser y la nada. Ensayo de ontología fenomenológica, Barcelona: Altaya.

Sartre, Jean Paul (1989), El existencialismo es un humanismo, Barcelona: Edhasa.

Sartre, Jean Paul (1947), «La liberté cartésienne», en Situations I, Paris: Gallimard, pp. 314-335.

Scott-Baumann, Alison (2009), Ricœur and the hermeneutics of suspicion, London, New York: Continuum.

Zima, Peter V. (2015), Subjectivity and Identity: Between Modernity and Postmodernity, London, New York: Bloomsbury. 